\title{
Chapter 3-Summary
}

In chapter 3, attention has been concentrated on the interrelationships between the enteric nervous system (ENS) and the sympathetic and parasympathetic systems, which transmit signals to the central nervous system (CNS). While up until now the ENS has been regarded as virtually autonomous-as a "little" or "second" brain which influences the gut locally - the evidence to substantiate this is based on inconclusive premises. The evidence presented in this session challenges this view and demonstrates considerable connection and interplay between the ENS and CNS.

Recent tracer and electrophysiological studies have shown, independently, that vagal efferents ramify extensively into a network of fibres which are in contact with large numbers of enteric neurones in the myenteric plexus of the stomach and proximal small intestine. Vagal afferents also ramify extensively, forming intraganglionic laminar endings in the enteric plexuses and intramuscular arrays which are in intimate contact with the interstitial cells of Cajal.

The sympathetic innervation of the gastrointestinal tract arises from abdominal prevertebral ganglia (PVG) - the coeliac, superior mesenteric, and inferior mesenteric ganglia. The ganglia serve as integrating centres between the CNS and ENS for regulation of intestinal motility, blood flow, and secretion. The recent identification of two messenger molecules which may mediate afferent input to PVG and modulate sympathetic efferent output has opened a door for the development of pharmacological tools. One of these messengers is carbon monoxide which is synthesised by haeme oxygenase 2 . In mice lacking this enzyme, gastrointestinal transit is markedly altered. By developing blockers of this enzyme, it should be possible to examine more precisely the role of carbon monoxide in gastrointestinal function. Another important messenger is the hormone leptin which acts on neurones in the hypothalamus to control food intake and energy balance. Immunochemistry has been used to study the location of leptin receptors and suggests that they are located intracellularly in PVG neurones. In view of their importance in food intake and energy utilisation, studies are currently being undertaken to examine the distribution of these receptors in PVG and enteric ganglia from obese and starved animals.

The sympathetic innervation to the gut influences blood flow, epithelial transport, motility, and endocrine cells. It has been shown that innervation of a major part of the gastrointestinal wall is scarce and that most adrenergic fibres make contact with the neurones of the enteric plexuses in the alimentary canal. However, there is also sympathetic innervation to precapillary and postcapillary blood vessels in the gut. Whereas in the case of intestinal motility and epithelial transport there is a link between sympathetic control and mechanical stimulation, vasodilatation evoked by mechanical stimulation is not influenced by activation of sympathetic vasoconstrictor fibres. Blood vessels are rather influenced by release of mediators such as 5-hydroxytryptamine (5-HT) from the intestinal mucosa, which in turn stimulate nerve terminals under the intestinal epithelium.

Studies performed on enterochromaffin cells suggest that release of hormones from endocrine cells in the gastrointestinal tract is under the influence of the autonomic nervous system; for example, 5-HT release is inhibited by stimulation with $\alpha$ adrenergic agonists and cholinergic stimulation has the opposite effect. Autonomic control of endocrine cells in the gastrointestinal tract may alter their sensitivity to luminal stimuli by influencing their membrane potential. By controlling the sensitivity of the luminal sensory receptors, it may be possible to influence gastrointestinal symptoms.

In conclusion, this chapter has illustrated the complex connections between the enteric neurones and the other extrinsic visceral afferent and autonomic pathways (both sympathetic and parasympathetic). The discovery of messenger molecules which might mediate afferent input and modulate sympathetic efferent output at the ganglion level and the finding that the sensitivity of endocrine cells can be influenced by autonomic input, has raised interesting possibilities for pharmacological intervention. 